\title{
Re: Managing dyslipidaemia for the primary prevention of cardiovascular disease
}

Rapid Response Letter in the BMJ 25 March 2018

Citation: Hippisley-Cox J. Re: Managing dyslipidaemia for the primary prevention of cardiovascular disease. British Medical Journal 360: k946 doi:10.1136/bmj.k946

[Rapid Response letter published online: 25 March 2018]

Ryan et al have recently written a useful article on managing dyslipidaemia for the primary prevention of cardiovascular disease1. They consider the evidence regarding the benefits and harms of statin medication and conclude that, "At present we have no way of knowing who will experience the benefits or harms of statin therapy before starting medication". We would like to highlight to readers a couple of our papers which have addressed this question2. In 2010, we published a large population-based cohort study using the QResearch database (www.qresearch.org) to individualise the risks of statins in men and women in England. We also produced a web calculator www.qintervention.org which allows an individual calculate these risks. In 2018, we published an update to the QDiabetes-2018 algorithm which, for the first time, quantifies the risk of developing diabetes among patients prescribing statins, taking account of their other risk factors3. The web calculator can be found here www.qdiabetes.org/2018. Lastly, we would also like to highlight the publication of QRISK3

https://qrisk.org/three/index.php last year in the BMJ4 which will replace QRISK2 later this year.

References:

1. Ryan A, Heath S, Cook P. Managing dyslipidaemia for the primary prevention of cardiovascular disease. BMJ 2018;360 doi: 10.1136/bmj.k946

2. Hippisley-Cox J, Coupland C. Individualising the risks of statins in men and women in England and Wales: population-based cohort study. Heart 2010;96(12):939-47. doi: 10.1136/hrt.2010.199034 [published Online First: 2010/05/22]

3. Hippisley-Cox J, Coupland C. Development and validation of QDiabetes-2018 risk prediction algorithm to estimate future risk of type 2 diabetes: cohort study. BMJ 2017;359 doi: 10.1136/bmj.j5019

4. Hippisley-Cox J, Coupland C, Brindle P. Development and validation of QRISK3 risk prediction algorithms to estimate future risk of cardiovascular disease: prospective cohort study. BMJ 2017;357 doi: 10.1136/bmj.j2099

Competing interests: JHC is professor of clinical epidemiology at the University of Nottingham and codirector of QResearch a not-for-profit organisation that is a joint partnership between the University of Nottingham and Egton Medical Information Systems (leading commercial supplier of IT for $55 \%$ of general practices in the UK). JHC is also a paid director of ClinRisk, which produces open and closed source software to ensure the reliable and updatable implementation of clinical risk algorithms within clinical computer systems to help improve patient care.

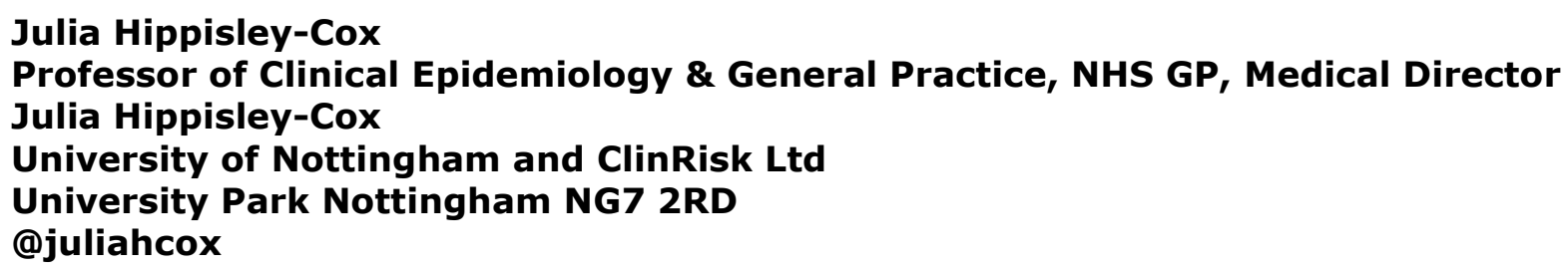

